\title{
Galactic wind shells and high redshift radio galaxies
}

\section{On the nature of associated absorbers}

\author{
M. Krause \\ Astrophysics Group, Cavendish Laboratory, Cambridge CB3 0HE, UK \\ e-mail: M.Krause@mrao.cam.ac.uk \\ Received 19 August 2004 / Accepted 15 March 2005

\begin{abstract}
A jet is simulated on the background of a galactic wind headed by a radiative bow shock. The wind shell, which is due to the radiative bow shock, is effectively destroyed by the impact of the jet cocoon, thanks to Rayleigh-Taylor instabilities. Associated strong HI absorption, and possibly also molecular emission, in high redshift radio galaxies which is observed preferentially in the smaller ones may be explained by that model, which is an improvement of an earlier radiative bow shock model. The model requires temperatures of $\approx 10^{6} \mathrm{~K}$ in the proto-clusters hosting these objects, and may be tested by high resolution spectroscopy of the Ly $\alpha$ line. The simulations show that - before destruction - the jet cocoon fills the wind shell entirely for a considerable time with intact absorption system. Therefore, radio imaging of sources smaller than the critical size should reveal the round central bubbles, if the model is correct.
\end{abstract}

Key words. hydrodynamics - instabilities - shock waves - galaxies: active - radio continuum: galaxies

\section{Introduction}

Radio galaxies at high redshift $(z \gtrsim 2)$ show huge (similar to the radio size), luminous $\left(\approx 10^{43}-10^{45} \mathrm{erg} / \mathrm{s}\right)$ Ly $\alpha$ halos (Röttgering et al. 1996; van Ojik et al. 1997; Reuland et al. 2003). Being the progenitors of today's brightest cluster galaxies (Carilli et al. 2001), they pinpoint proto-clusters of galaxies (Venemans et al. 2002, 2003). Many of the systems smaller than $\approx 50 \mathrm{kpc}$ show associated absorption, preferentially on the blue wing of the emission line (van Ojik et al. 1997; Binette et al. 2000; De Breuck et al. 2000). While the low column density absorbers $\left(N_{\mathrm{HI}} \approx 10^{13}-10^{14} \mathrm{~cm}^{-2}\right)$ are probably mainly due to the Ly $\alpha$ forest, the high column density ones $\left(N_{\mathrm{HI}} \approx 10^{18}-10^{20} \mathrm{~cm}^{-2}\right)$ are found much more frequently than expected from the Ly $\alpha$ forest and hence belong to the radio galaxy (van Ojik et al. 1997; Wilman et al. 2004). High resolution spectroscopy has revealed that some of the latter are made up of overlapping smaller ones. But some others have been confirmed (Wilman et al. 2003, 2004).

The frequency of this phenomenon points to common circumstances in the surroundings of these most massive objects in the young universe. A sound understanding of the emission line structure therefore promises insight into their assembly. Here, I present hydrodynamic simulations of a jet born inside a galactic wind shell, the latter having a radiative bow shock. This can be regarded as a modification of an earlier model (Krause 2002), where the jet itself produced the radiative bow shock. The big absorption systems might be identified with such a geometrically thin, dense shell, as I will discuss in the following.

Section 2 reviews the available models from the literature. Section 3 introduces the new one. The computation is presented in Sect. 4, and the implications and observational tests are discussed in Sect. 5.

\section{Review of absorber models}

\section{1. $10^{12}$ clouds in an ionisation cone}

In this model, proposed by van Ojik et al. $(1997), \approx 10^{12}$ clouds with a density of $n_{\mathrm{c}} \approx 100 \mathrm{~cm}^{-3}$, about $10^{8} M_{\odot}$ in total, are distributed throughout the region occupied by the radio galaxy. A central quasar, hidden from the observer by a central gas and dust torus, ionises the clouds within two cones, where the gas is also stirred up by interaction with the jet (observations indicate a typical line width of $1000 \mathrm{~km} \mathrm{~s}^{-1}$ ). Outside the cone, the clouds are neither ionised by the quasar nor stirred up by the radio source, and hence are able to absorb the Ly $\alpha$ emission of the ionised clouds in a narrower velocity range (typically $10-100 \mathrm{~km} \mathrm{~s}^{-1}$ ). The smaller sources are located in denser environments, which also produce more HI absorption.

This model cannot explain the tendency of these absorption systems to be blueshifted $\left(\approx 100-200 \mathrm{~km} \mathrm{~s}^{-1}\right)$. In at least one case the absorber has been detected in CIV (Binette et al. 2000), who inferred a lower metallicity in the absorber than in 
the emitting plasma. This would require that the absorbing gas is physically distinct from the line emitting plasma.

\subsection{Extended low density shell}

Binette et al. (2000) also infered a high ionisation of the absorbing gas. The lack of an obvious source of the ionising photons forced them to conclude that the shell was a low density quiescent absorbing screen surrounding the radio galaxy. They favoured a density of $10^{-3}-10^{-2} \mathrm{~cm}^{-3}$, because such an absorber would be ionised by the metagalactic background radiation. They proposed an evolutionary scenario: once the radio source reaches the screen, the increased pressure compresses the denser parts, that from now on can only be seen when inside the ionisation cone, while the rest gets completely ionised.

This scenario also has problems with the velocity structure. Suppose there would be a quiescent gas halo at a temperature of a few times $T=10^{4} \mathrm{~K}$ and a density of $n=10^{-3} \mathrm{~cm}^{-3}$ surrounding the centre of the radio galaxy in a distance of $\approx 50 \mathrm{kpc}$. The cooling time would be $t_{\mathrm{c}}=k_{\mathrm{B}} T / n \Lambda \approx$ Myr, where $\Lambda \approx 10^{-22} \mathrm{erg} \mathrm{cm} / \mathrm{s}$ is the cooling function (e.g. Sutherland \& Dopita 1993). After that time, which is much shorter than the expected age of the observed radio sources, a cooling flow should be established, and the absorbers should appear on the red wing, not on the blue one, as observed. It was concluded that the absorbing gas has to be outflowing due to a galactic wind (Wilman et al. 2003). In this case, the density distribution would be expected to be smooth $\left(\approx r^{-2}\right)$. When the source expands, it should gradually reduce the neutral column. This has not been shown to be able to accommodate the observed bimodal distribution of column densities. Another problem arises from the temperature in such an outflow, which is given by

$$
\begin{aligned}
T= & \frac{(\gamma-1) L t}{(4 / 3) \pi r^{3} n k_{\mathrm{B}}}=10^{7} \mathrm{~K} \\
& \times \frac{L}{10^{42} \mathrm{erg} / \mathrm{s}} \frac{t}{100 \mathrm{Myr}} \frac{10^{-3} \mathrm{~cm}^{-3}}{n}\left(\frac{25 \mathrm{kpc}}{r}\right)^{3},
\end{aligned}
$$

where $\gamma$ is the adiabatic index, $L$ is the driving power of the galactic wind, a few supernovae per century, $r$ is the radius of the shell, and $t$ the time for which the wind is active. So, one would expect the gas in these outflows to be too hot to contain neutral hydrogen. If they should contain denser condensations, that provide the absorption, the ionisation problem reappears. If the parameters of these winds would be tuned to the right temperature, the cooling flow problem reappears. The gas would be expected to loose its pressure support and switch from outflow to inflow.

\subsection{Jet with radiative bow shock}

Three consequences arise if a jet propagates into a dense medium. First, the bow shock slows down. Second, it may become radiative, and third, very light jet physics applies (Krause 2002, 2003). In this case, the bow shock is first spherical, following the same law of motion as the stellar (and galactic) wind bubble, which reads for constant external density $\rho$ :

$$
\begin{aligned}
& r_{\mathrm{sh}}=12 \mathrm{kpc}\left(\frac{L}{10^{46} \mathrm{erg} / \mathrm{s}} \frac{m_{\mathrm{p}} \mathrm{cm}^{-3}}{\rho}\left(\frac{t}{10 \mathrm{Myr}}\right)^{3}\right)^{1 / 5} \\
& v_{\mathrm{sh}}=450 \mathrm{~km} \mathrm{~s}^{-1}\left(\frac{L}{10^{46} \mathrm{erg} / \mathrm{s}} \frac{m_{\mathrm{p} \mathrm{cm}^{-3}}}{\rho}\left(\frac{25 \mathrm{kpc}}{r_{\mathrm{sh}}}\right)^{2}\right)^{1 / 3} .
\end{aligned}
$$

An external density of a few $m_{\mathrm{p}} \mathrm{cm}^{-3}$ would slow the bow shock velocities down to the observed values. Such a slow bow shock would heat a surrounding X-ray atmosphere only marginally. The cooling time due to bremsstrahlung is $t_{\mathrm{c}}=$ $12 \operatorname{Myr} \sqrt{T / 10^{7} \mathrm{~K}} /\left(n / \mathrm{cm}^{-3}\right)$. Hence, if the density is high enough to slow down the bow shock as required, the bow shock will be radiative, even if the atmosphere has already keV temperatures. A thin and dense shell forms, that may provide the required column density. Since the shell is subject to a number of instabilities, the shell fragments, and the radiation can be seen unabsorbed through the holes, in the larger sources.

The natural column density of the shell, $N_{\mathrm{sh}}=$ $10^{23} \mathrm{~cm}^{-2}\left(\rho / 5 m_{\mathrm{p}} \mathrm{cm}^{-3}\right)\left(r_{\mathrm{sh}} / 25 \mathrm{kpc}\right)$, which would correspond to a surprisingly large gas mass of order $10^{13} M_{\odot}$, and is much more than observed. However, the shell clumps quickly, and a clumpy multi-phase medium is also what is infered from detailed simulations of $2 \mathrm{D}$ radiative shocks (Sutherland et al. 2003). In fact this may be the only way to explain that not only atomic hydrogen and highly ionised carbon, but also molecular gas is observed at the same velocities (De Breuck et al. 2003a,b). The effective column will be reduced. The low column regions might be ionised by the recently discovered inverse Compton (X-ray) emission of the cosmic microwave background from the radio cocoon, which may well extend into the UV (Scharf et al. 2003).

Another issue is the Ly $\alpha$ luminosity of the shell, which should be considerably below that of the emission line. Assuming one recombination per proton and a power law atmosphere $\rho=\rho_{0}\left(r_{\mathrm{sh}} / r_{0}\right)^{\kappa}$, this is given by:

$$
\begin{aligned}
L_{\mathrm{sh}, \mathrm{Ly} \alpha}= & 4 \pi r_{\mathrm{sh}}^{2} v_{\mathrm{sh}} h v_{\mathrm{Ly} \alpha} \rho / m_{\mathrm{p}} \\
= & \left(\frac{h v_{\mathrm{Ly} \alpha}}{m_{\mathrm{p}}}\right)\left[\frac{144(\kappa+3)}{\pi^{2}(\kappa+5)^{2}} L \rho_{0}^{2} r_{0}^{4}\left(\frac{r_{\mathrm{sh}}}{r_{0}}\right)^{4+2 \kappa}\right]^{1 / 3} \\
\approx & 3 \times 10^{43} \mathrm{erg} / \mathrm{s}\left[\frac{L}{10^{46} \mathrm{erg} / \mathrm{s}}\left(\frac{\rho_{0}}{5 m_{\mathrm{p}} \mathrm{cm}^{-3}}\right)^{2}\right. \\
& \left.\times\left(\frac{r_{0}}{25 \mathrm{kpc}}\right)^{4}\left(\frac{r_{\mathrm{sh}}}{r_{0}}\right)^{4+2 \kappa}\right]^{1 / 3} .
\end{aligned}
$$

The observational requirements may be fulfilled, postulating a high density inside of $\approx 10 \mathrm{kpc}$, and a steep decline $(\kappa<$ -2) further out. Clearly, the atmospheres have to be carefully tuned. Recent estimates on jet powers easily reach $10^{47} \mathrm{erg} / \mathrm{s}$ (Ghisellini 2003; Krause \& Camenzind 2003). Because high redshift radio galaxies are among the most powerful jet sources, this makes the model more difficult. 


\section{Jet within a galactic wind's radiative bow shock}

This model involves a galactic wind due to supernova activity in the host galaxy, probably related to a starburst. Due to the low power of such a wind compared to a typical jet power, it will only have an observational effect, if the wind starts long before the jet activity. The wind would have a radiative bow shock, which is responsible for the aforementioned Ly $\alpha$ absorption in the case of small jets. When the jets reach the shell, they destroy it so that the larger sources are no longer absorbed. The details of this process are studied in the simulation below.

In such a model, the parameters can be well constrained from observations. For the postshock gas to have a shorter cooling time than the preshock gas, the Mach number should be below six. This restricts the temperature of the atmosphere via the sound speed. A shock velocity of $200 \mathrm{~km} \mathrm{~s}^{-1}$, implies an X-ray atmosphere with $0.2-1.5 \times 10^{6} \mathrm{~K}$, in agreement with the non-detection of thermal bremsstrahlung in 4C 41.17 (Scharf et al. 2003). The age of the galactic wind bubble would be $t_{\mathrm{d}}=3 r_{\mathrm{sh}} / 5 v_{\mathrm{sh}}=73 \mathrm{Myr}\left(r_{\mathrm{sh}} / 25 \mathrm{kpc}\right)\left(200 \mathrm{~km} \mathrm{~s}^{-1} / v_{\mathrm{sh}}\right)$. This is to exceed the cooling time due to bremsstrahlung, and consequently the pre-wind density is limited to $n \gtrsim$ $0.06 \mathrm{~cm}^{-3} \sqrt{T / 10^{6} \mathrm{~K}}\left(25 \mathrm{kpc} / r_{\mathrm{sh}}\right)\left(v_{\mathrm{sh}} / 200 \mathrm{~km} \mathrm{~s}^{-1}\right)$. It could be even lower, if the cooling is to happen in the postshock gas only, and more realistic cooling functions are applied. Such a density is similar to local clusters of galaxies. The resulting total absorbing column in the postshock gas would be two orders of magnitude below that of the previous model, alleviating the above described problem, but still requiring the dominant fraction not to be HI. The power required to drive the wind is: $L>5 \times 10^{43} \mathrm{erg} / \mathrm{s} \sqrt{T / 10^{6} \mathrm{~K}}\left(v_{\mathrm{sh}} / 200 \mathrm{~km} \mathrm{~s}^{-1}\right)^{4}\left(r_{\mathrm{sh}} / 25 \mathrm{kpc}\right)$, equivalent to some supernovae per year. Starbursts in otherwise normal galaxies at high redshift show winds with similar parameters (Dawson et al. 2002; Pettini et al. 2002; Tapken et al. 2004).

\section{Simulation}

\subsection{Setup}

The jet inside a galactic wind scenario was simulated in 2.5D with the hydrodynamic code NIRVANA (Ziegler \& Yorke 1997). To the evolution equations for mass, momentum and energy, the force of a dark matter halo, and the zero metal, zero field cooling function from Sutherland \& Dopita (1993) was added. The number densities of electrons and ions, which determine the cooling rate, are computed from the total density and temperature, in the approximation of a pure hydrogen plasma in collisional ionisation equilibrium. The computational domain spanned $[Z \times R]=[200 \mathrm{kpc} \times 100 \mathrm{kpc}]$, resolved by [2046 $\times 1022]$ cells. A control run was performed at double resolution, but spanned only $50 \mathrm{kpc}$ radially, which the first simulation showed to be sufficient. The grid was initialised with an isothermal King atmosphere $(\rho=$ $\left.0.3 m_{\mathrm{p}} \mathrm{cm}^{-3} /\left(1+r^{2} / 100 \mathrm{kpc}^{2}\right), r^{2}=R^{2}+Z^{2}\right)$ at a temperature of $10^{6} \mathrm{~K}$. In order to break the symmetry, random fluctuations on the percent level have been added to the density. The galactic wind was simulated by a distributed energy and mass density
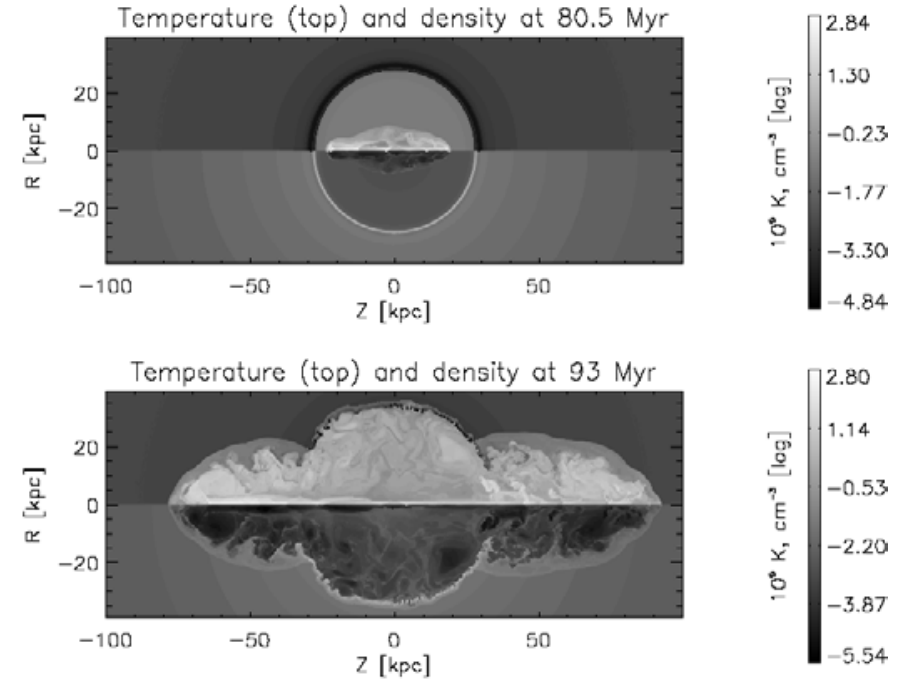

Fig. 1. Density and temperature distribution after $80.5 \mathrm{Myr}(0.5 \mathrm{Myr}$ after jet start), $93 \mathrm{Myr}$ (13 Myr after jet start) for the low resolution simulation.
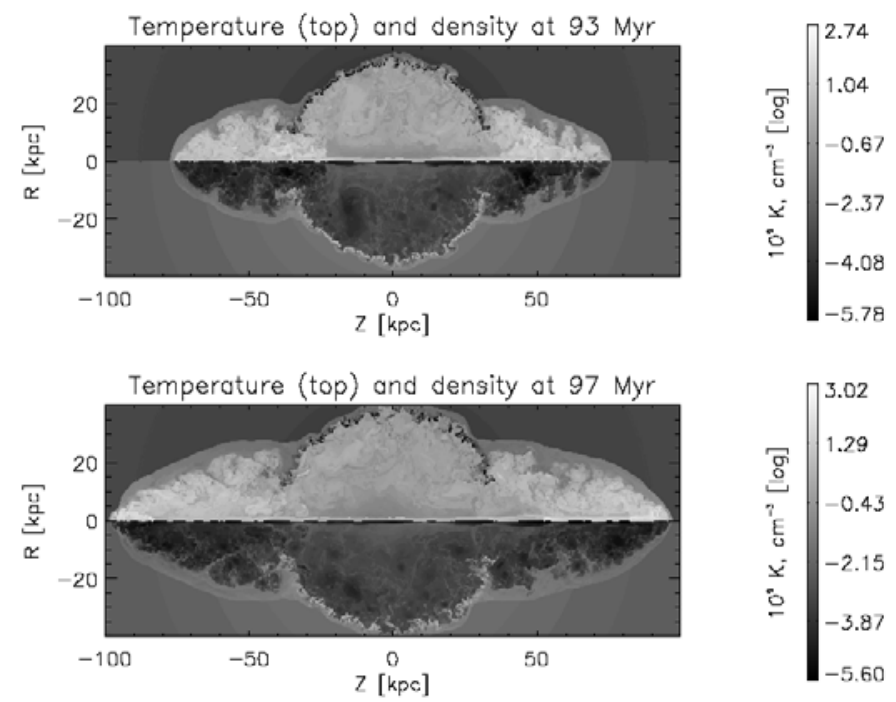

Fig. 2. Density and temperature distribution after $93 \mathrm{Myr}$ (13 Myr after jet start), $97 \mathrm{Myr}$ (17 Myr after jet start) for the high resolution simulation. Only part of the grid is shown in the radial direction. Comparison to Fig. 1 shows the same overall evolution. Details that differ include the more evident asymmetry in the low resolution simulation, and the slower growth of the outer jet bubbles in the high resolution case.

increase, $\propto \exp (-r / 3 \mathrm{kpc}), 3 \times 10^{43} \mathrm{erg} / \mathrm{s}$, and $10 M_{\odot} / \mathrm{yr}$ in total. With these parameters, both cold gas due to a cooling flow and a radiative bow shock by the galactic wind can be expected. After $80 \mathrm{Myr}$, a bipolar jet with a radius of $1 \mathrm{kpc}$, a density of $10^{-5} \mathrm{~m}_{\mathrm{p} \mathrm{cm}} \mathrm{cm}^{-3}$, a Mach number of 13 , a velocity of $2 / 3$ the speed of light, and a total power of $4 \times 10^{45} \mathrm{erg} / \mathrm{s}$ was injected in the centre of the grid.

\subsection{Results}

Density and temperature distributions at $80.5 \mathrm{Myr}$ and $93 \mathrm{Myr}$ are shown in Fig. 1 (low resolution simulation) and at $93 \mathrm{Myr}$ and 97 Myr in Fig. 2 (high resolution simulation). 

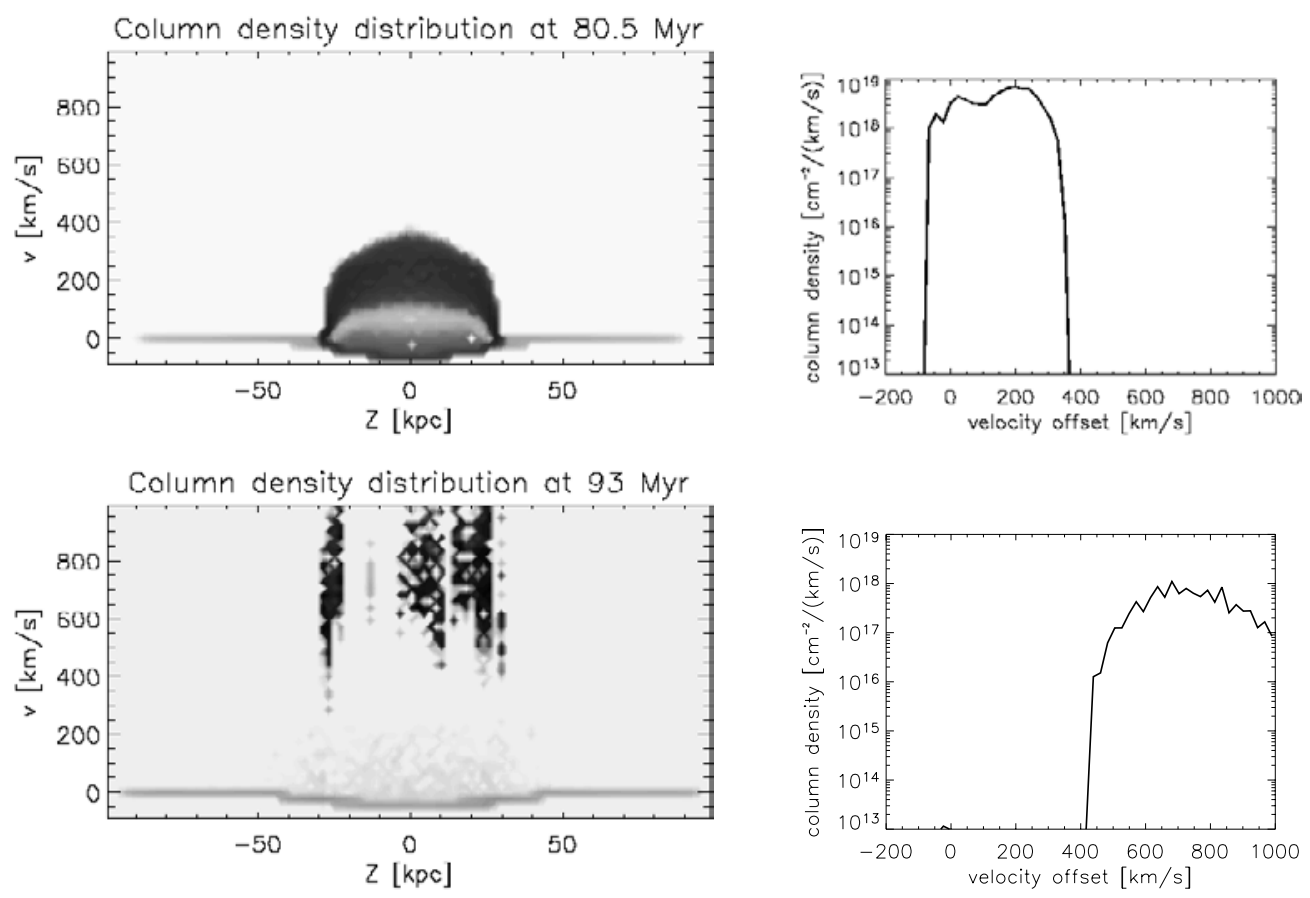

Fig. 3. Column density of neutral hydrogen over Z-position and radial velocity (left), and integrated over space (right), after $80.5 \mathrm{Myr}$ (top, $0.5 \mathrm{Myr}$ after jet start) and $93 \mathrm{Myr}$ (bottom) for the low resolution simulation.

During the first $80 \mathrm{Myr}$, the galactic wind establishes a radiative bow shock, which runs into a cooling flow atmosphere. The radiative bow shock is marginally resolved, i.e. the temperature rises in the shock, and then cools to $\approx 10^{4} \mathrm{~K}$, where the cooling function was cut. Up to $\approx 81 \mathrm{Myr}$, the jet stays inside of the wind shell. Then it quickly bores holes at the heads, and the cocoon fills up the wind bubble. The increased pressure inside the wind bubble now accelerates the shell, which is then destroyed due to Rayleigh-Taylor instabilities.

In this phase, large streams of gas, raining down from the shell into the interior of the bubble can be observed (Figs. 2 and 8). Being stirred up by the jet's backflow, such gas may contribute to emission line gas. In the simulation, even at the higher resolution, this gas soon mixes with the very hot and rarefied plasma from the jet cocoon, but in reality it may stay unspoiled at emission line temperatures, contributing to the emission line halo. Even so, the entrained gas condenses at the lower parts of the cocoon next to the jet beam (Fig. 6), due to the background gravity. The galactic wind also contributes somewhat to the high density in that region. Also, the gas is constantly stirred up due to backflowing vortices.

The wind shell fragments into dense clumps. Hotter gas flows past these clumps and establishes a new, faster and nonradiative bow shock. A closeup of the shell fragments can be seen in Fig. 8. The maximum density, which is reached in the shell, is shown over time in Fig. 6 (right). For the high resolution simulation, it starts to rise at $81 \mathrm{Myr}$, when the tip of the jet hits the shell. Oscillating, it then keeps rising as long as the shell remains intact. In that phase, the jet cocoon compresses gas into the wind shell. At about $90 \mathrm{Myr}$, the fragmentation starts. The cocoon material can then escape from the bubble, the pressure drops, and the gas clouds expand again. The jet leaves the grid shortly after 97 Myr. Data after that time could be affected by that. At lower resolution, the same things happen, but take more time. At jet start and towards the end of the simulation, the maximum density is about half the one in the highly resolved simulation. This reflects the resolution increase by a factor of two. In between, the higher resolution simulation reaches a four times higher density than the low resolution one. The simulation is obviously not numerically converged. Nonetheless, even if not fully converged, the simulations serve to illustrate the fundamental physical behaviour that is important in understanding the associated absorber phenomenon, namely that the jet-wind interaction causes the formation of many dense clouds through the operation of the R-T instability. Furthermore, there is good reason to apply this kind of resolution, which effectively cuts the R-T modes smaller than some critical wavelength, as explained below.

The column density distribution for neutral hydrogen has been analysed at $80.5 \mathrm{Myr}$ and $93 \mathrm{Myr}$ (low resolution, Fig. 3) and 93 Myr and 97 Myr (high resolution, Fig. 4). The neutral fraction has been calculated according to collisional ionisation equilibrium (Sutherland \& Dopita 1993). The 2D representations (over $Z$-position and $R$-velocity) show that, as long as the jet is inside the wind bubble, both, the cooling flow (down to $\left.-100 \mathrm{~km} \mathrm{~s}^{-1}\right)$ and the wind shell $\left(200 \mathrm{~km} \mathrm{~s}^{-1}-400 \mathrm{~km} \mathrm{~s}^{-1}\right)$ are able to form significant absorption systems, totally covering the radio galaxy. The infalling material has a very narrow velocity distribution. For the wind shell, the full width at half maximum is $\approx 100 \mathrm{~km} \mathrm{~s}^{-1}$. The highest column density is found between $200 \mathrm{~km} \mathrm{~s}^{-1}$ and $250 \mathrm{~km} \mathrm{~s}^{-1}$. The speed of the radiative shock is $200 \mathrm{~km} \mathrm{~s}^{-1}$. At $93 \mathrm{Myr}$, the shell has been destroyed. At low resolution, no significant column is left below $500 \mathrm{~km} \mathrm{~s}^{-1}$, neither of the wind shell nor of the cooling flow. The surviving clumps still produce high column, but are not coherent, neither in space nor in velocity space. At higher resolution, 

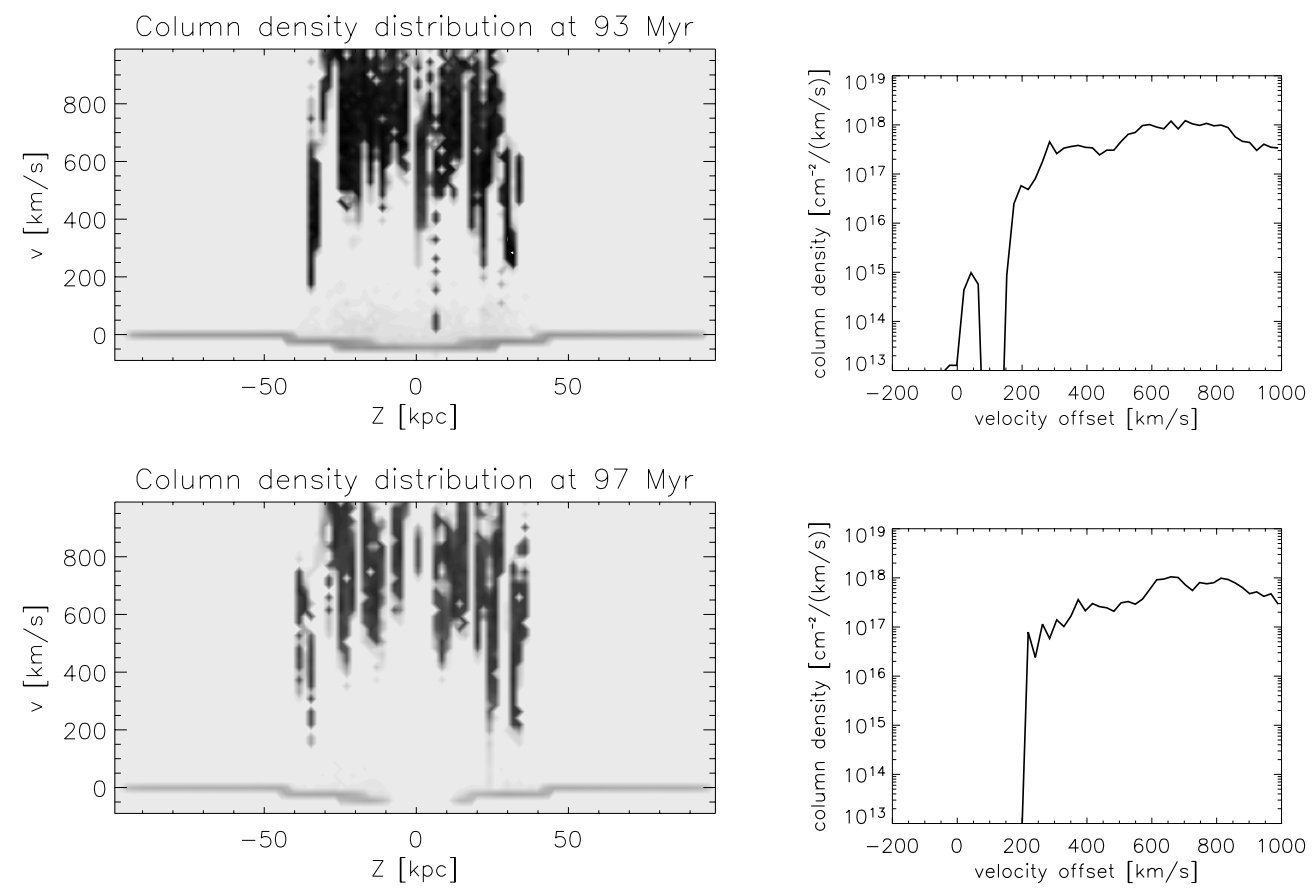

Fig. 4. Column density of neutral hydrogen over Z-position and radial velocity (left), and integrated over space (right), after 93 Myr (top, $13 \mathrm{Myr}$ after jet start) and $97 \mathrm{Myr}$ (bottom) for the high resolution simulation.
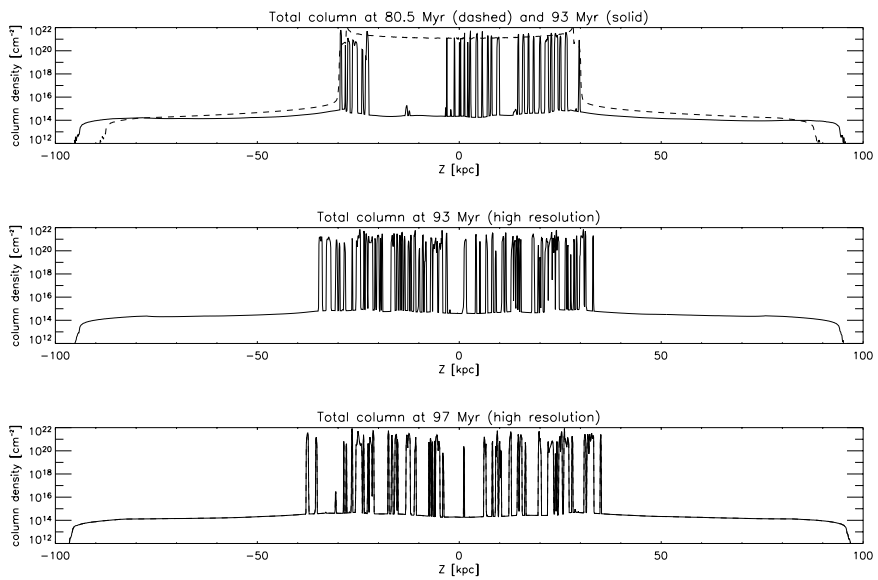

Fig. 5. Column over axial position (top: low resolution, dashed line: 80.5 Myr, solid line: $93 \mathrm{Myr}$; middle: high resolution, $93 \mathrm{Myr}$; bottom: high resolution, $97 \mathrm{Myr})$.

the clouds are more resilent. There is significant column left between $200 \mathrm{~km} \mathrm{~s}^{-1}$ and $400 \mathrm{~km} \mathrm{~s}^{-1}$, also at late times. However, this comes mainly from non-central regions, and at $97 \mathrm{Myr}$ the central regions are devoid of slow neutral hydrogen.

At that time, the covering fraction is $\approx 20 \%$. It takes $\approx 1-3$ Myr for a significant drop (Figs. 5 and 7). At higher resolution, the covering fraction starts to drop later. It then drops quickly to $50 \%$, after which it flattens, ending in a slightly higher number than the low resolution simulation.

\section{Discussion}

Using a galactic wind as the driver of the radiative shock reduces the lower limit on the external density to values observed in present day galaxy clusters. Also, the Ly $\alpha$ self luminosity (3) of the shell is low, as required for absorption systems. The jet head pierced the wind shell after 1 Myr. The simulation showed that the shell is able to totally cover the inner $50 \mathrm{kpc}$ of the jet with high column for $7 \mathrm{Myr}$ after jet start. At that time the jet is considerably more extended than the wind shell. This means that the real wind shell would be smaller than assumed here, since the radio jets are observed to have diameters of $50 \mathrm{kpc}$ when the absorption disappears.

The jet impact accelerated the absorbing shell to a higher velocity region, the shell thereby fragmented due to the Rayleigh-Taylor instability, and part of the material fell into the interior of the now radio cocoon filled shell. During this process the covering fraction reduced to $\approx 20 \%$. The simulations were $2 \mathrm{D}$ axisymmetric. In reality, the reduction along the third dimension should be similar, which would result in a final true covering fraction of about $20 \%^{2} \approx 4 \%$. The emission lines could pass nearly unchanged through such a region.

The assumption of axisymmetry also affects other parts of the simulation. General considerations for jet simulations are valid also in this case (Norman 1993), i.e. jets are less stable in 3D simulations, and magnetic fields as well as a high Mach number may be required for stable propagation over the observed distances. An interesting extra is the interaction of the cocoon with the wind shell fragments. This may well cause asymmetries in the vortex shedding process, leading to the bending of the jet. This is observed in some of the larger sources (e.g. van Ojik et al. 1996; Pentericci et al. 1997, 1998). Therefore, further investigation is necessary.

The total neutral column is still higher than observed. The reason may be that a significant fraction of the gas cools down even further to form molecular hydrogen (compare Dopita \& Sutherland 2003, p 338). When appropriately resolved, 

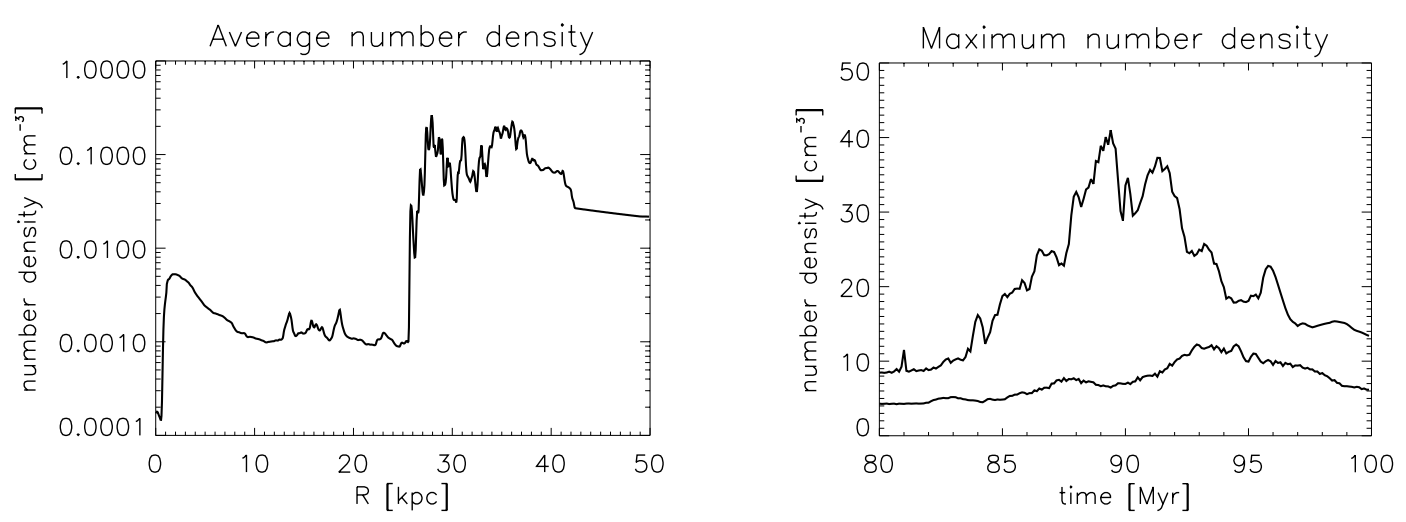

Fig. 6. Left: average number density within the shell $(\mathrm{abs}(Z)<28 \mathrm{kpc})$ at $97 \mathrm{Myr}$ for the high resolution simulation. Right: maximum density, always occuring in the shell, over time. The high resolution simulation takes the higher density values.
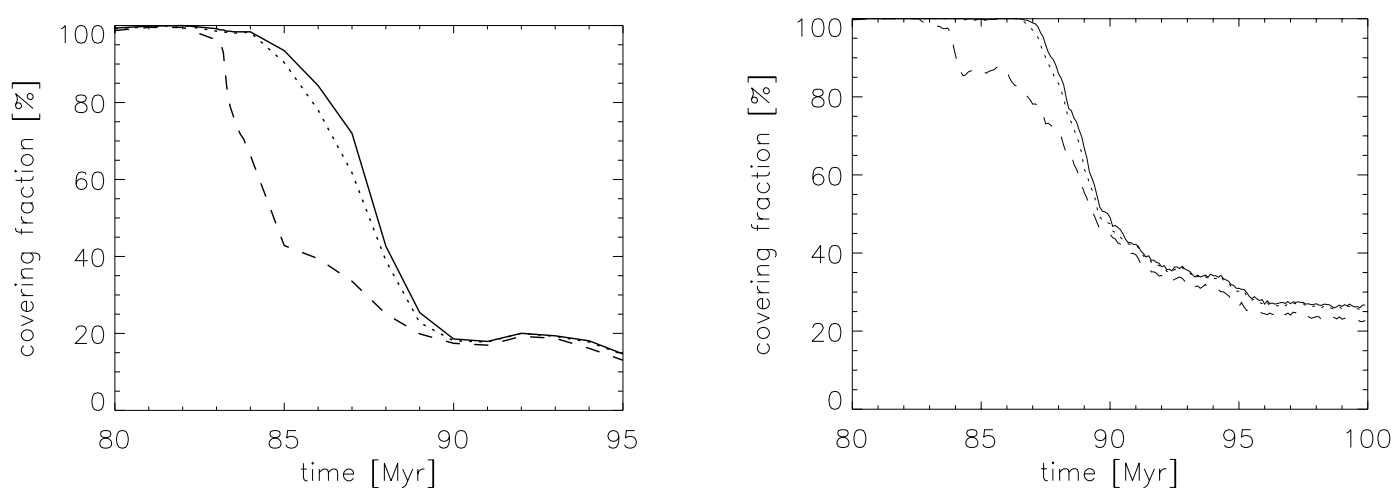

Fig. 7. Covering fraction of neutral hydrogen within $|Z|<30 \mathrm{kpc}$ for a column in excess of $10^{18} \mathrm{~cm}^{-2}$ (solid), $10^{19} \mathrm{~cm}^{-2}$ (dotted), and $10^{20} \mathrm{~cm}^{-2}$ (dashed). The left (right) panel shows the result for the low (high) resolution simulation.

radiative shocks have recently been shown to become turbulent due to the thermal instability (Sutherland et al. 2003). Various ionisation species as well as molecular hydrogen might be present. Details would require more involved shock models than presently available.

There are few examples of partially covered Ly $\alpha$ regions in the literature. TN J1338-1942 might be a rare example (De Breuck et al. 1999). The higher velocity clumps, present after shell destruction, may explain complex velocity profiles of larger sources.

Doubling of the resolution raised the density in the shell by more than a factor of two. The shell fragments are more resilent at higher resolution (compare Mellema et al. 2002), and therefore covering fraction and neutral column at low velocities reduce more slowly. The fragmentation of the shell starts earlier at higher resolution. This is in agreement with the linear growth time for the Rayleigh-Taylor instability, which is proportional to the wavelength, i.e. it starts faster if smaller wavelengths are resolved. The simulation is therefore not converged. Convergence would be achieved by processes not included in the simulation. The wind shell would be supported by its magnetic field, limiting its density $n_{\mathrm{s}}$, and the Rayleigh-Taylor instability would finally be stabilised at the critical wavelength $\lambda_{\mathrm{c}}$ by the transversal magnetic field $B$ (Jun et al. 1995) of the radio cocoon:

$\lambda_{\mathrm{c}}=20 \mathrm{pc}\left(\frac{B}{10^{-5} \mathrm{G}}\right)^{2}\left(\frac{10^{-7} \mathrm{~cm} / \mathrm{s}^{2}}{g}\right)\left(\frac{10 \mathrm{~cm}^{-3}}{n_{\mathrm{s}}}\right)$.

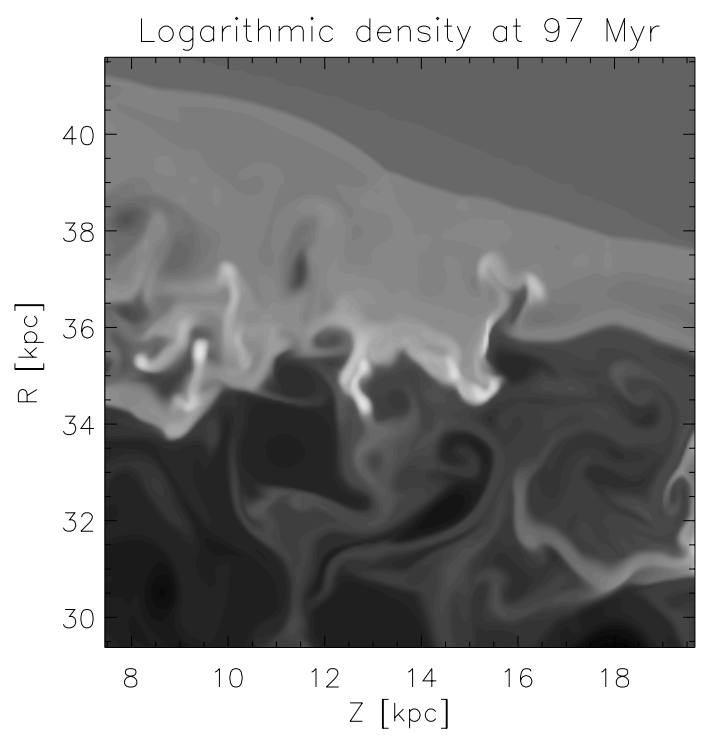

Fig. 8. Closeup of the shell fragments at $97 \mathrm{Myr}$ for the high resolution simulation.

Here, $g$ is a typical shell acceleration of $500 \mathrm{~km} \mathrm{~s}^{-1}$ in $10 \mathrm{Myr}$. Depending on these parameters, the shell also may fragment faster or slower in individual sources. For these reasons, the applied resolutions seem to be reasonable.

The simulation also shows that the blueshift of the absorber changes with position by $\approx 1 \mathrm{~km} \mathrm{~s}^{-1} \mathrm{kpc}^{-1}$. This is tiny, but 
might be detected in high resolution spectra. For sources near the critical size, high quality radio observations at low frequencies may reveal the round central cocoon structure that is produced by the jet wind interaction. Such observations may provide a test of the thin shell model.

Acknowledgements. This work was supported by the Deutsche Forschungsgemeinschaft (SFB 439). The computations have been carried out at the HLRS in Stuttgart (Germany).

\section{References}

Binette, L., Kurk, J. D., Villar-Martín, M., \& Röttgering, H. J. A. 2000, A\&A, 356, 23

Carilli, C. L., Miley, G., Röttgering, H. J. A., et al. 2001, in Gas and Galaxy Evolution, ed. J. E. Hibbard, M. Rupen, \& J. H. van Gorkom, San Francisco, ASP Conf. Proc., 240

Dawson, S., Spinrad, H., Stern, D., et al. 2002, ApJ, 570, 92

De Breuck, C., van Breugel, W., Minniti, D., et al. 1999, A\&A, 352, L51

De Breuck, C., Röttgering, H., Miley, G., van Breugel, W., \& Best, P. 2000, A\&A, 362, 519

De Breuck, C., Neri, R., Morganti, R., et al. 2003a, A\&A, 401, 911

De Breuck, C., Neri, R., \& Omont, A. 2003b, New Astron. Rev., 47, 285

Dopita, M. A., \& Sutherland, R. S. 2003, Astrophysics of the diffuse universe (Berlin, New York: Springer), Astronomy and astrophysics library, ISBN 3540433627

Ghisellini, G. 2003, New Astron. Rev., 47, 411

Jun, B., Norman, M. L., \& Stone, J. M. 1995, ApJ, 453, 332

Krause, M. 2002, A\&A, 386, L1

Krause, M. 2003, A\&A, 398, 113

Krause, M., \& Camenzind, M. 2003, New Astron. Rev., 47, 573
Mellema, G., Kurk, J. D., \& Röttgering, H. J. A. 2002, A\&A, 395, L13

Norman, M. L. 1993, in Space Telescope Science Institute Symposium Series, Proc. of the Astrophysical Jets Meeting, held in Baltimore 1992 May 12-14, ed. D. Burgarella, M. Livio, \& C. P. O’Dea (Cambridge, UK: Cambridge University Press)

Pentericci, L., Roettgering, H. J. A., Miley, G. K., Carilli, C. L., \& McCarthy, P. 1997, A\&A, 326, 580

Pentericci, L., Roettgering, H. J. A., Miley, G. K., et al. 1998, ApJ, 504, 139

Pettini, M., Rix, S. A., Steidel, C. C., et al. 2002, ApJ, 569, 742

Reuland, M., van Breugel, W., Röttgering, H., et al. 2003, ApJ, 592, 755

Röttgering, H., Miley, G., \& van Ojik, R. 1996, The Messenger, 83, 26

Scharf, C., Smail, I., Ivison, R., et al. 2003, ApJ, 596, 105

Sutherland, R. S., Bicknell, G. V., \& Dopita, M. A. 2003, ApJ, 591, 238

Sutherland, R. S., \& Dopita, M. A. 1993, ApJS, 88, 253

Tapken, C., Appenzeller, I., Mehlert, D., Noll, S., \& Richling, S. 2004, A\&A, 416, L1

van Ojik, R., Röttgering, H. J. A., Carilli, C. L., et al. 1996, A\&A, 313,25

van Ojik, R., Röttgering, H. J. A., Miley, G. K., \& Hunstead, R. W. 1997, A\&A, 317, 358

Venemans, B., Miley, G., Kurk, J., Rottgering, H., \& Pentericci, L. 2003, The Messenger, 111, 36

Venemans, B. P., Kurk, J. D., Miley, G. K., et al. 2002, ApJ, 569, L11

Wilman, R. J., Jarvis, M. J., Röttgering, H. J. A., \& Binette, L. 2003, New Astron. Rev., 47, 279

Wilman, R. J., Jarvis, M. J., Röttgering, H. J. A., \& Binette, L. 2004, MNRAS, 351, 1109

Ziegler, U., \& Yorke, H. W. 1997, Computer Physics Commun., 101, 54 